\title{
Analysis of texture features for wood defect classification
}

\author{
Nur Dalila Abdullah', Ummi Raba'ah Hashim², Sabrina Ahmad ${ }^{3}$, Lizawati Salahuddin ${ }^{4}$ \\ ${ }^{1}$ Department of Information Technology and Communication, Politeknik Muadzam Shah, Malaysia \\ ${ }^{2,3,4}$ Centre for Advanced Computing Technology, Fakulti Teknologi Maklumat dan Komunikasi, Universiti Teknikal \\ Malaysia Melaka (UTeM), Malaysia
}

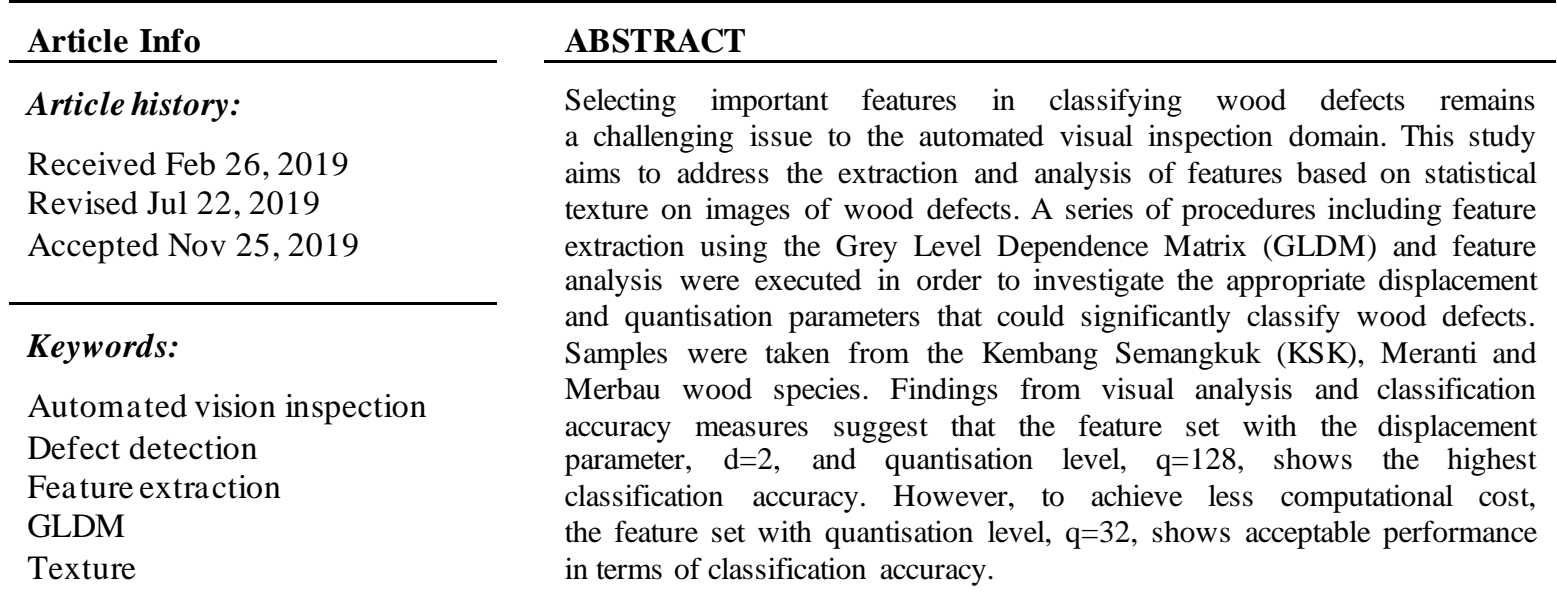

This is an open access article under the CC BY-SA license.

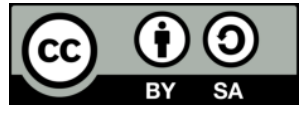

\section{Corresponding Author:}

Nur Dalila Abdullah,

Faculty of Information Technology and Communication,

Politeknik Muadzam Shah,

LebuhrayaTunRazak, 26700 MuadzamShah, Pahang, Malaysia.

Email: dalilaabdullah@yahoo.com

\section{INTRODUCTION}

Wood is classified as a natural biological material and it is easily attacked by microorganisms [1]. These microorganisms can destroy the wood structure and allow the presence of defect on the wood surface, thus also affecting the quality of the wood [2]. Poor quality will lead to unsatisfactory and unappealing wooden products should this defect material be used. The presence of defects in wooden material reduces its value. In order to avoid this from happening, the manufacturer should take preventive measure where they should check and determine the presence of defect on the wood surface. These wood defects can be detected manually using visual inspection. The manual inspection could lead to human error and this is affected depending on the skill level, experience of the worker and alertness. However it takes a lot of time and the process is slow [3]. Due to this problem, it is difficult to ensure that a complete inspection had been done and the results and quality control could be unreliable. Many studies and research had been done in order to improve the process of detecting wood defects. One of the solutions is to use a machine vision based inspection system which can save inspection time and indirectly the results can lead to a reliable quality control process [3].

AVI is an automated tool that controls the quality of the manufacturer products. It needs to be setup and the automation process will follow certain standards that are strictly set by the manufacturer. By having AVI in the wood industry, it can increase the production line by inspecting a large number of products without having the human limitations such as tiredness, boredom, lack of knowledge, and lack 
of training. When doing an inspection using AVI, the hardware, such as a camera, is connected to a computer. It also needs to consider integration with other aspects which are the lighting, working practices and how to handle the equipment. The AVI system works by the computer controlling the camera, lighting, and handling system. The camera will analyse the image captured using an image processing routine and afterwards the handling system will perform the appropriate action. The images will be displayed on the Visual Display Unit (VDU).

The features extraction is one of the ways to detect a defect on the timber surface. Once the features are extracted, it could lead to another step which classifies them into different defect classes but this resea rch is focused on the process of features extraction only. The defect on each of the timber surface is not equal in size and shape. The extraction is not only done on the defect surface but also on clear wood to differentiate whether the timber surface has defects or not. Therefore, it is very crucial to determine and generate a quality feature set in order to find the right features to be used to detect a defect. By selecting the optimum set of features, the detection process could be performed with high accuracy and reliability [4].

The purpose of this study is to contribute to a part of the process in developing an automated vision inspection which is the feature extraction technique using the Grey Level Dependence Matrix (GLDM) for wood defect classification. By using this feature extraction technique, it can lead to an analysis of the most appropriate displacement and quantisation parameters of GLDM. As an addition, the techniques also shows the simplicity of the feature extraction, easy comparability computing and the statistical features show good directional qualities [5]. This study also could contribute to generate a feature set of timber defect and this will make it easier to detect the defect on the timber surface.

\section{RESEARCH METHOD}

This section explains in detail the research methodology for the proposed texture feature technique which is GLDM. This chapter further discusses the research design which includes the operational framework and previous studies on wood defect detection. The operational framework discusses the procedure flow on how to perform the GLDM technique. The previous studies will be discussing on the texture that every article emphasize in their work.

\subsection{Operational framework}

The operational framework in Figure 1 shows the proposed approach to determine the feature extraction set in characterizing the wood defects. Firstly, the orientation of spatialdependence matrix was set, and the features were extracted with various displacement and quantisation parameters. The data source u sed in this study is from the Universiti Teknikal Melaka Malaysia (UTeM) wood defect database [6]. There are nine defect types from four wood species, namely Rubberwood, Kembang Semangkuk (KSK), Merbau and Meranti. Example of images from the data source are displayed in Table 1. Next, the extracted features were further analysed to investigate the appropriate displacement and quantization setting for GLDM. Finally, the performance of the features extracted were measured using visual exploratory analysis and classification accuracy.

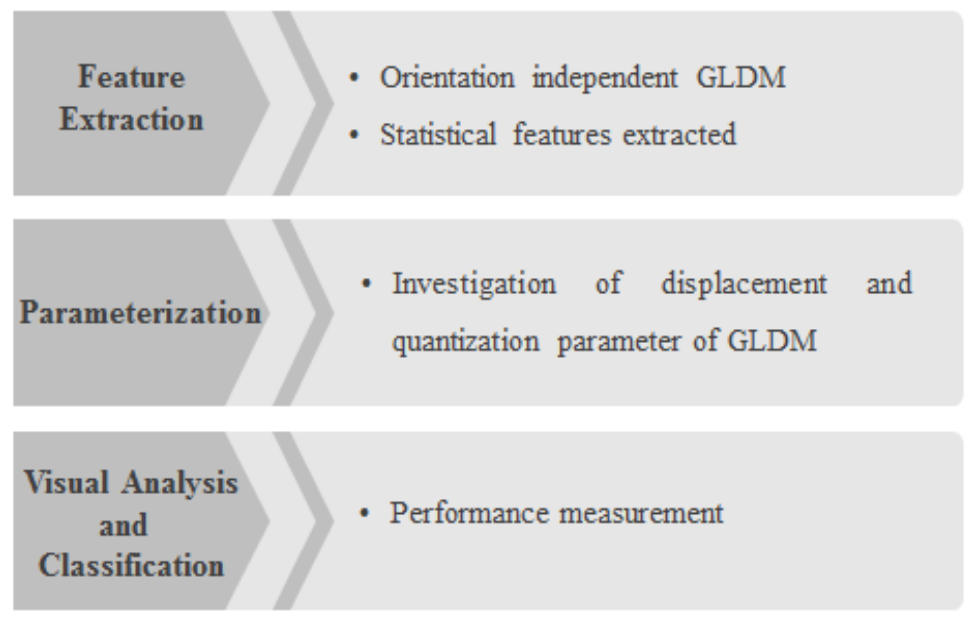

Figure 1. Proposed approach to determine the feature extraction 
Table 1. Example of images of 9 defect types [6]

\begin{tabular}{|c|c|c|c|c|c|}
\hline Defect & Image & Defect & Image & Defect & Image \\
\hline Knot & 0 & Bark Pocket & $p$ & Blue Stain & \\
\hline $\begin{array}{l}\text { Surface } \\
\text { Check }\end{array}$ & & Borer Holes & $t$ & Wane & \\
\hline Brown Stain & 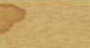 & Rot & & Split & \\
\hline
\end{tabular}

\subsection{Previous studies on wood defect detection}

Many researches had been done on finding an appropriate feature extraction technique for wood defect classification. It is supported that texture is the most favourable features to be used for the purpose of feature extraction method [7]. In a study, the Grey Level Co-occurrence Matrix (GLCM) technique was used and the dataset is taken from the University of Oulu, Finland. The data set consisted of 90 images with $51 \times 48$ resolutions. The features used in this study were contrast, correlation, energy and homogeneity. The maximum and minimum values were calculated for contrasting data [3].

In another work, Hashim et al. focused on three features which are energy, contrast and correlation based on spatial dependence matrix. The spatial dependence matrix is parameterized with distance $=1$ and image quantisation at 64 intensity levels. It shows that the energy feature value for clear wood is higher compared to the defect image which means clear wood has a higher homogeneity [8]. Hashim et al. further extended their work with 20 statistical features from Grey Level Dependence Matrix (GLDM) and demonstrated an acceptable classification accuracy across multiple wood species [9]. Hittawe et al. proposed the use of LBP and SURF features extraction in his study. The training dataset contained 50 images of knot and 50 images of cracks. The technique extracts a set of low-level features and builds a visual vocabulary by quantisation of the features [10].

Meanwhile, Mahram, Shayesteh and Jafarpour proposed three feature extraction techniques which are GLCM, LBP and Statistical moments. The GLCM method calculates co-occurrence matrix at four rotation directions $\left(0^{\circ}, 45^{\circ}, 90^{\circ}\right.$, and $\left.135^{\circ}\right)$ and fifteen texture features were computed, which are as follows: mean, energy, entropy, variance, dissimilarity, contrast, correlation, inverse difference moment, cluster shade, cluster prominence, sum average, sum variance, sum entropy, and difference entropy [11]. From the previous studies, it shows that there is no unique solution to represent defect features. Every study chooses a different set of feature extraction technique depending on the problem and dataset used. Table 2 summarised feature extraction technique used in previous wood defect detection or classification studies.

Table 2. Feature extraction technique used in previous wood defect detection or classification studies

\begin{tabular}{|c|c|c|}
\hline Feature Extraction Techniques & Texture Features & References \\
\hline \multirow[t]{6}{*}{$(\mathrm{GLCM})$} & 20 statistical features & [9] \\
\hline & $\begin{array}{l}5 \text { features: Angular Second Moment, Contrast, Correlation, Entropy, } \\
\text { Inverse Difference Moment }\end{array}$ & [14] \\
\hline & $\begin{array}{l}15 \text { features: Mean, Energy, Entropy, Variance, Dis similarity, Contrast, } \\
\text { Correlation, Inverse Difference Moment, Cluster Shade, Cluster } \\
\text { Prominence, Sum Average, Sum Variance, Sum Entropy, and } \\
\text { Difference entropy }\end{array}$ & [11] \\
\hline & 4 features: Energy, Entropy, Homogeneity, Inverse Difference Moment & {$[5]$} \\
\hline & 4 features: Not mentioned & [15] \\
\hline & $\begin{array}{l}5 \text { features: Angular second moment, Contrast, Correlation, Entropy, } \\
\text { Variance }\end{array}$ & [16] \\
\hline Statistical moment & Geometrical moment of order & [11] \\
\hline $\begin{array}{l}\text { Image block percentile colour histogram } \\
\text { and eigenvector texture feature }\end{array}$ & $\mathrm{k}$-max eigenvectors as image block & {$[6]$} \\
\hline SURF & Low-level feature & [10] \\
\hline Tamura texture & $\begin{array}{l}6 \text { features: Coarseness, contrast, directionality, linearity, regularity and } \\
\text { roughness }\end{array}$ & [16] \\
\hline Independent component analysis (ICA) & FastICA Algorithm(Negentrophy) & [19] \\
\hline
\end{tabular}




\section{RESULTS AND DISCUSSION}

Figure 2 illustrates the procedures to extract statistical texture features using the GLDM approach. The first step was to convert all the sub-images into greyscale images. Subsequently, GLDM were constructed at various displacement value $(\mathrm{d}=1 \ldots 30)$ and quantisation level $(\mathrm{q}=8,16,32,64,128,256)$. The generated GLDM from previously selected parameter was generated for all four orientations which are $0^{\circ}, 45^{\circ}, 90^{\circ}$, and $135^{\circ}$. Twenty statistical feature values were extracted and normalized from the GLDM. Next, the data is normalized to plot a graph showing feature values for every displacement and quantisation case in order to find the most appropriate para meters for GLDM.

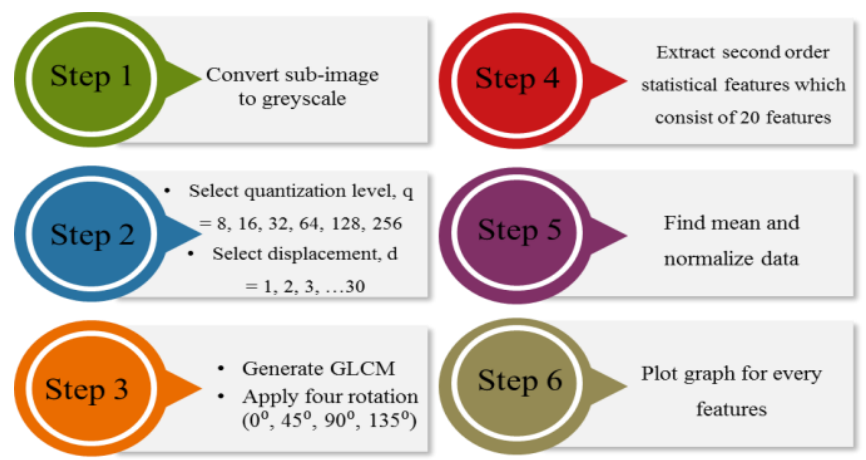

Figure 2. Procedures to extract statistical texture features

\subsection{Parameter analysis of GLDM}

The feature extraction was repeated at various displacement and quantisation levels which produced 150 datasets, and for each of these datasets the mean of the normalized data was calculated to compare the feature values across displacement values and quantisation levels. The displacement chosen was up until 30 as it is half of the image size which is $60 \times 60$. Figure 3 shows graph of normalized feature values across displacement and quantisation levels for knots defect from KSK species. Only few features are shown in the figures which are cluster prominence, cluster shade, contrast and correlation.
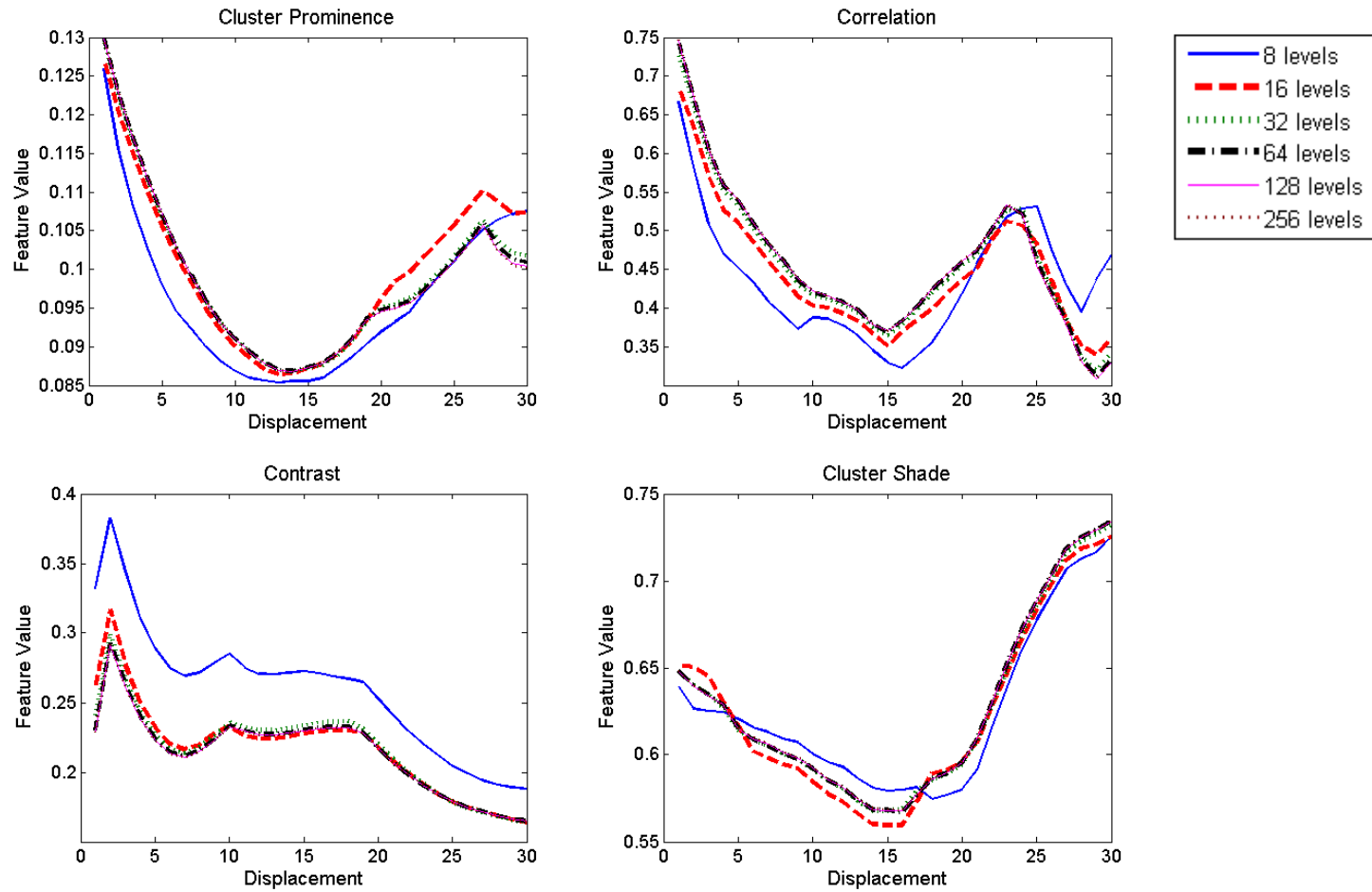

Figure 3. Normalized feature values for KSK species (defect=knots) 
From the observation of the plotted graph, the curves patterns do not have much change across different quantisation levels for 20 features. The similarities of upward and downward slopes indicate that the quantisation level does not affect the extracted texture properties. Even though some of the slopes are slightly different in degree, it still preserves the displacement curve. However, the quantisation level of 8 and 16 differ from other quantisation levels. This is possibly due to the loss of informa tion whenever the image is reduced to a lower resolution. Meanwhile, the higher quantisation level shows that the curves are closely similar to each other and it is hard to distinguish between the curves. For later performance comparison, the suggested quantisation level, therefore must be higher than $q=16$, which are $\mathrm{q}=32,64,128,256$.

The upward or downward trend of the graph shows a consistent pattern for all features up to displacement, $d<=5$. All graph started to show changes in the slope direction when it reached displacement, $d>5$. From the observation, it can be concluded that the texture property began to have a structural change whenever a higher displacement is applied. Petrou and Sevilla [20] emphasized that a high displacement value is most suitable for capturing textures of large primitive patterns while the lower displacement fits for textural with detailed patterns. This study is focused on the detailed patterns. Hence, for later performance analysis, it is suggested that the most appropria te displacement should be less than $5(\mathrm{~d}<=5)$.

\subsection{Feature range analysis}

In feature range analysis, we selected 35 samples from each defect type for two species which consisted of 630 dataset. Subsequently, the dataset will be used to generate a graph to discriminate between defect and clear wood. The graph will be generated according to the displacement, $d<=5$ and quantisation level, q=32, 64 and 128. Each dataset needs to be normalized in the range of 0 to 1 in order to plot the feature range graph. Figure 4 shows graph plotted for two features which are correlation and contrast extracted at $\mathrm{d}=5$ and $\mathrm{q}=32$ for KSK species across multiple defects. From the graphs, it is noticeable that clear wood shows a clear distinction where the curves deviate from other defect classes. Meanwhile, the other defects are overlapping and clustered. This give us an indication that features extracted at parameter $\mathrm{d}<5$ and $\mathrm{q}=32$ is sufficient to separate between defect and clearwood classes.
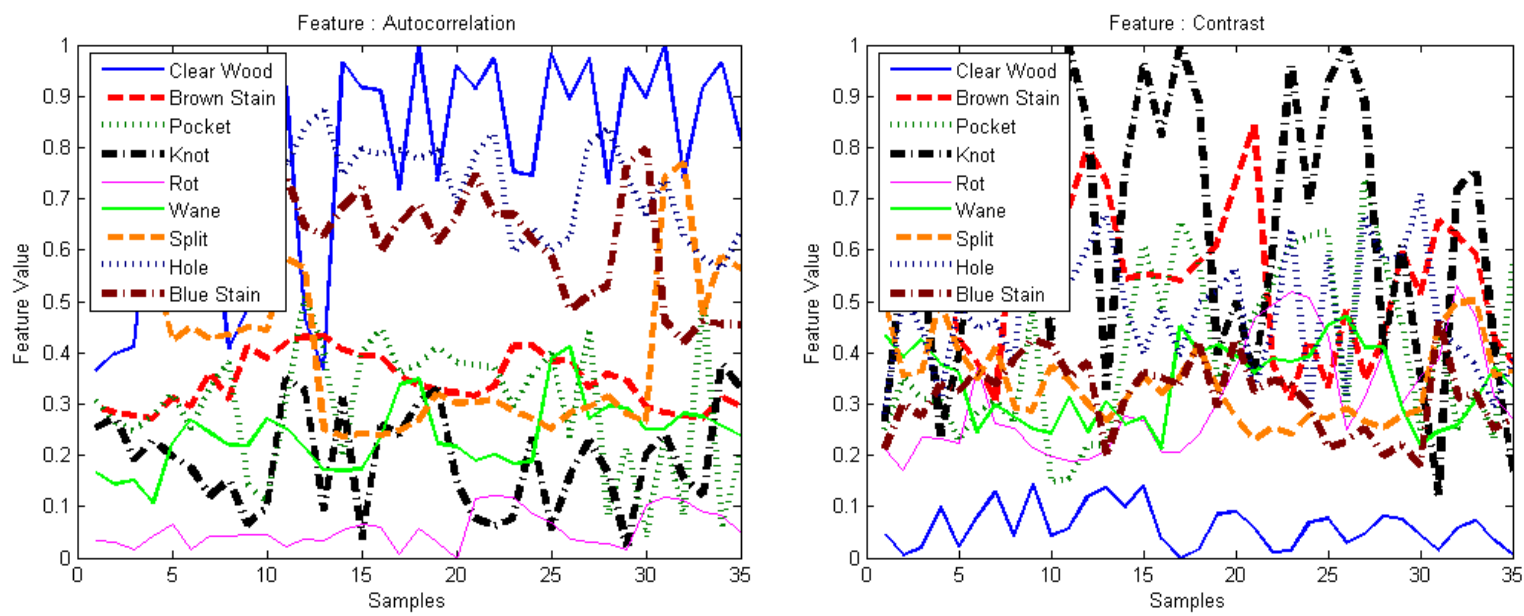

Figure 4 . KSK species $(q=32, d=5)$

\subsection{Classification performance}

From the previous section, we concluded that the displacement parameter is at its best at $\mathrm{d}=1,2,3,4,5$ because there is no significant change in slope when the displacement is less 5 . For quantisation level, it is most appropriate at quantisation level, $\mathrm{q}=32,64,128$ because the curves are closely similar to each other. Feature range analyses also have shown promising result in discriminating between defect and clear wood classes. Next, we further investigated the feature performance using classification accuracy measure. The normalized features for KSK and Merbau were used in the experiment. We compared the classification accuracy (F-measure) of the feature set across various quantisation and displacement parameters using the classification performance of a standard classifier which is the Artificial Neural Network (ANN). 
The experiment was run across multiple species, namely Merbau, KSK and Meranti. The dataset for Meranti was taken from Hashim et al. [21]. The experiment was run at displacement, $\mathrm{d}=1,2,3,4,5$ and quantisation level, q=32, 64, 128 for each species, whereby each dataset contained nine defects including clear wood. For this classification experiment, we performed a cross-validation of 10 folds and ran the experiment at 20 repetitions. Table 3 shows the classification accuracy (F-measure) across species at different quantisation and displacement parameter.

Table 3. F-measure across species

\begin{tabular}{ccccc}
\hline Quantisation, $q$ & Displacement, $d$ & KSK & Merbau & Meranti \\
\hline 32 & 1 & 0.85 & 0.90 & 0.83 \\
32 & 2 & 0.85 & 0.86 & 0.88 \\
32 & 3 & 0.92. & 0.85 & 0.88 \\
32 & 4 & 0.89 & 0.91 & 0.88 \\
32 & 5 & 0.84 & 0.94 & 0.87 \\
64 & 1 & 0.85 & 0.92 & 0.83 \\
64 & 2 & 0.83 & 0.87 & 0.86 \\
64 & 3 & 0.89 & 0.87 & 0.86 \\
64 & 4 & 0.89 & 0.92 & 0.88 \\
64 & 5 & 0.92 & 0.91 & 0.85 \\
128 & 1 & 0.85 & 0.91 & 0.83 \\
128 & 2 & 0.86 & 0.85 & 0.86 \\
128 & 3 & 0.87 & 0.90 & 0.86 \\
128 & 4 & 0.93 & 0.91 & 0.86 \\
128 & 5 & 0.92 & 0.95 & 0.83 \\
\hline
\end{tabular}

From the experiments, it reveals that all datasets have a score of 0.83 and above although the performance slightly varied across species and also across various displacement and quantisation parameters. This clearly shows that lower quantisation does not affect the performance of the classification, therefore, lower quantisation does not indicate loss of information [22, 23]. This is further supported by Ma et al. [24], where it is claimed that lower quantisation level decreases the computational load wh ile not affecting the discriminatory power of GLDM. Although higher quantisation will improve both accuracy and separability, lower quantisation will aid in decreasing computational load. The classification can still be successful even if the quantisation level is reduced. Figure 5 further shows the pictorial representation of independent orientation GLDM for blue stain defect at different displacement and quantisation levels. It can be observed that, even at varying quantisation level, the GLDM still ma naged to capture the same pattern for the defect. However, when applying a higher quantisation level, the matrix image becomes clearer and more precise.

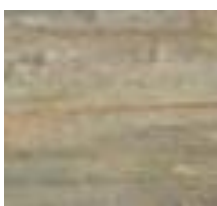

Original image

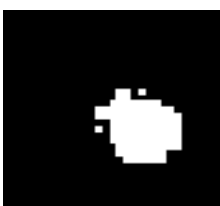

$Q=32, D=2$

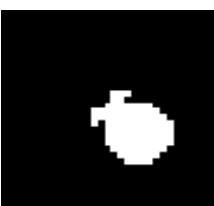

$Q=32, D=4$

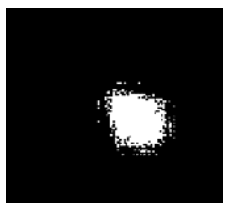

$Q=128, D=2$

Figure 5. GLDM pictorial representation (species=KSK, defect=bluestain)

\section{CONCLUSION}

In this study, we aim to investigate on how to discriminate each type of wood defect using GLDM feature extraction technique and we worked on experiments in determining the significant feature set using visual analysis and classification measures. The research was divided into two phases, where the first phase extracted the feature from an image sample and the second phase used the feature extract from the first phase to experiment using the classification measures [25]. The first phase began with the first step which was to convert the image into grayscale image and select the parameter for four orientations which are $0^{\circ}, 45^{\circ}, 90^{\circ}$, and $135^{\circ}$. The mean and normalized data were calculated from the feature extracted and a graph of normalized feature values against displacement and quantisation levels was plotted. From the graph, we can observe the trends and reduce the number of quantisation levels to be run in the experiment. It is summarize $d$ 
that the best displacement are at $\mathrm{d}<5$ and quantisation level, $\mathrm{q}=32,64,128,256$. However, quantisation, $q=256$ was excluded from the experiment due to the image itself which was at quantisation level of 256.

In the feature range analysis, a plotted graph was generated to distinguish between defect and clear wood. The feature value of energy, correlation and contrast can be used as a guideline to discriminate between defect and clear wood. The second phase used the classification measures to measure the accuracy of which parameters that are most suited to be used in timber defect detection. To determine the most appropriate parameter to be selected is by comparing the F-measure across different datasets used in the experiment. The experiment had 45 datasets, where each 15 datasets were for three different species (KSK, Merbau and Meranti). Each dataset had 35 samples for each defect which resulted in 315 samples for each dataset. The total of samples used was 14175. From the investigation of the experiment, it can be resolved that the utmost parameters can be used is displacement, $\mathrm{d}=2$ and quantisation, $\mathrm{q}=128$. However, if one wants a less computational load and cost, it is worthwhile to select the displacement, $\mathrm{d}=2$ and quantisation, $q=32$ or displacement, $d=4$ and quantisation, $q=32$. It is finally concluded that although higher quantisation will improve both accuracy and separability, lower quantisation will aid in decreasing computationalload.

Therefore, the classification of wood defect can still be successful even if the quantisation level is reduced. To conclude the research objectives, which include the two phases mentioned above have been accomplished. Including a feature extraction using the Grey Level Dependence Matrix (GLDM) technique, analysing the appropriate displacement and quantisation level from the feature extraction and lastly identification of a significant feature set of timber defects using visual analysis and classification mea sures.

\section{ACKNOWLEDGEMENTS}

This research is supported by Universiti Teknikal Malaysia Melaka (UTeM) under UTeM Short Term Research Grant (PJP/2018/FTMK(3B)/S01630).

\section{REFERENCES}

[1] M. Hongbo, Q. Dawei, H. Nan, and M. Zhang, "Edge extraction of wood image with rot based on gray transformation," Int. Conf. Comput. Appl. Syst. Model., pp. 605-608, 2010.

[2] F. Iwani, M. Redzuan, and M. Yusoff, "Knots timber detection and classification with c-support vector machine," Bulletin of Electrical Engineering and Informatics, vol. 8, no. 1, pp. 246-252, 2019.

[3] R. Qayyum, K. Kamal, T. Zafar, and S. Mathavan, "Wood defects classification using GLCM based features and PSO trained neural network," 22nd Int. Conf. Autom. Comput. (ICAC), pp. 3-7, 2016.

[4] M. S. Packianather and B. Kapoor, "A wrapper-based feature selection approach using bees algorithm for a wood defect classification system,” 10th Syst. Syst. Eng. Conf., pp. 498-503, 2015.

[5] R. Athilakshmi, W. Amitabh, and B. Nagarajan, "Defect identification of lumber through correlation technique with statistical and textural feature extraction method," in International Conference on Communication and Computational Intelligence, pp. 524-528, 2010.

[6] W. Song, T. Chen, Z. Gu, W. Gai, W. Huang, and B. Wang, "Wood materials defects detection using image block percentile color histogram and eigenvector texture feature," Int. Conf. Inf. Sci. Mach. Mater. Energy (ICISMME), pp. 780-784, 2015.

[7] S. A. Dhole and R. P. Shaikh, "Review of leaf unhealthy region detection using image processing techniques," Bulletin of Electrical Engineering and Informatics, vol. 5, no. 4, pp. 451-453, 2016.

[8] U. R. Hashim, A. K. Muda, S. Z. Hashim, and M. B. Bonab, "Rotation invariant texture feature based on spatial dependence matrix for timber defect detection," in 13th International Conference on Intelligent Systems Design and Applications (ISDA), pp. 307-312, 2013.

[9] U. R. Hashim, S. Z. Hashim, and A. K. Muda, "Performance evaluation of multivariate texture descriptor for classification of timber defect," J. Light Electron Opt., vol. 127, no. 15, pp. 6071-6080, 2016.

[10] M. M. Hittawe, S. M. Muddamsetty, D. Sidibe, and F. Meriaudeau, "Multiple features extraction for timber defects detection and classification using SVM," in 2015 IEEE International Conference on Image Processing (ICIP), pp. 427-431, Dec 2015.

[11] A. Mahram, M. G. Shayesteh, and S. Jafarpour, "Classification of wood surface defects with hybrid usage of statistical and textural features," in 2012 35th International Conference on Telecommunications and Signal Processing, pp. 749-752, 2012.

[12] A. Fahrurozi, S. Madenda, Ernastuti, and D. Kerami, "Wood texture features extraction by using GLCM combined with various edge detection methods," Journal of Physics: Conference Series, vol. 725, no. 1, pp. 1-11, 2016.

[13] P. Cavalin, L. S. Oliveira, A. L. Koerich, and A. S. Britto, "Wood defect detection using grayscale images and an optimized feature set," IECON 2006-32nd Annual Conference on IEEE Industrial Electronics, Paris, pp. 3408-3412, 2006.

[14] M. Khalid, E. Lew Yi Lee, R. Yusof, and M. Nadaraj, "Design of an intelligent wood species recognition system," International Journal of Simulation: Systems, Science and Technology, vol. 9, no. 3, pp. 9-19, 2008. 
[15] P. Barmpoutis, I. Barboutis, and P. Lefakis, "Detection of various characteristics on wooden surfaces, using scanner and image processing techniques," in 27th International Conference on Wood Modification and Tehnology, no. 2013, pp. 7-13, 2016.

[16] X. Yonghua and W. Jin-cong, "Optik study on the identification of the wood surface defects based on texture features \&,” Opt.-Int. J. Light Electron Opt., vol. 126, no. 19, pp. 2231-2235, 2015.

[17] Y. X. Zhang, Y. Q. Zhao, Y. Liu, L. Q. Jiang, and Z. W. Chen, "Identification of wood defects based on LBP features," 2016 35th Chinese Control Conference (CCC), Chengdu, pp. 4202-4205, 2016.

[18] S. J. Elias et al., "Face recognition attendance system using local binary pattern (LBP)," Bulletin of Electrical Engineering and Informatics, vol. 8, no. 1, pp. 239-245, 2019.

[19] A. M. Mansour, J. Abukhait, and I. Zyout, "Speed sign recognition using independent component analysis," Int. J. Electrc,. Electron., \& Comput. Syst., vol. 17, no. 1, pp. 1-7, Nov 2013.

[20] M. Petrou and P. G. Sevilla, "Image Processing: Dealing with Texture," Wiley, 2006.

[21] U. R. Hashim, S. Z. Hashim, and A. K. Muda, "Image collection for non-segmenting approach of timber surface defect detection," Int. J. Adv. Soft Comput. Appl., vol. 7, no. 1, pp. 15-34, 2015.

[22] L.-.Soh and C. Tsatsoulis, "Texture analysis of SAR sea ice imagery using gray level co-occurrence matrices," in IEEE Transactions on Geoscience and Remote Sensing, vol. 37, no. 2, pp. 780-795, March 1999.

[23] D. A. Clausi, "An analysis of co-occurrence texture statistics as a function of grey level quantization," Can. J. Remote Sens., vol. 28, no. 1, pp. 45-62, 2002.

[24] J. Ma, Z. Zhang, C. Wang, and Z. Chen, "Classifier-based feature fusion for texture discrimination," Proc. 2009 Int. Conf. Inf. Eng. Comput. Sci. ICIECS, pp. 1-4, 2009.

[25] A. Shawkat and S. A. Kate, "Improving support vector machine generalisation via input space normalisation," Int.. J. Electr. Electron. Comput. Syst., June 2011. 